\title{
Reduction of the Impact of Group Delay Ripples in 10-Gb/s NRZ Grating-Compensated Dispersion-Managed fiber System Using Nonlinear Optical Loop-Mirrors
}

\author{
A. Labruyère ${ }^{\dagger}$ K. Nakkeeran, ${ }^{\ddagger}$ Y. H. C. Kwan, ${ }^{\dagger}$ \\ and P. K. A. Wai ${ }^{\dagger}$ \\ ${ }^{\dagger}$ Photonics Research Centre and Department of Electronic and Information \\ Engineering, The Hong Kong Polytechnic University, Hung Hom, Hong Kong \\ $\ddagger$ Department of Engineering, Fraser Noble Building, King’s College, University of \\ Aberdeen, Aberdeen AB24 3UE, UK \\ Phone: +852 2766-6197, fax: +852 2362-8439, email: enlabruy@polyu.edu.hk
}

\begin{abstract}
We investigate the re-amplification and reshaping using nonlinear optical loopmirrors in a 10-Gb/s non-return-to-zero grating-compensated dispersion-managed transmission line with standard fibers. A large improvement of the system performance is achieved.
\end{abstract}

\section{Introduction}

Lumped dispersion compensation technique based on chirped fiber gratings (CFGs) have been suggested as the most economic and simple strategy to upgrade the installed terrestrial fiber transmission systems. However, CFGs have group delay ripples (GDR) due to the imperfections in the manufacturing process. In return-to-zero (RZ) grating-compensated dispersion-managed (DM) fiber systems, GDR induces the growth of side peaks and induces timing-jitter [1], while in non-return-to-zero (NRZ) grating-compensated DM fiber systems, GDR is responsible for a large eye opening penalty [2]. Recently, using the nonlinear optical loopmirrors (NOLMs), the suppression of the growth of side peaks in RZ grating-compensated DM fiber systems have been demonstrated theoretically [1]. In fact, lumped regeneration techniques based on NOLMs have been widely investigated in the last few years $[3,4]$. But those investigations were limited to high-speed RZ transmissions. In this work, we investigate the use of lumped NOLMs together with lumped dispersion compensation based on CFGs in NRZ transmission line. We show that the transmission penalty induced by GDR in a 10-Gb/s grating-compensated DM fiber system is dramatically reduced under the action of NOLMs.

\section{Model}

We assume ripples having a sinusoidal profile with period $2 \pi / T_{0}$ and amplitude $\Gamma / T_{0}$. Hence, the GDR can be written as $t_{g}(\omega)=\left(\Gamma / T_{0}\right) \cos \left(\omega T_{0}+\theta\right)$, where $\theta$ denotes the relative phase between signal carrier frequency and the ripples. It is well known that the signal will be distorted by multiple side pulses with exponentially decreasing amplitude in the temporal domain [2]. The first pulses are located at $t= \pm n T_{0}$ where $n$ is an integer. The side pulses may either contribute to the accumulation of radiations in the 'zeroes', or interfere with the 'ones' of the signal. In that case, the resulting interference profile depends on the local field and the worst case is obtained when $\theta=0$ or $\pi$ as the contrast is maximum. Here, we show that the impact of GDR over multiple CFGs during propagation can be reduced by inserting NOLMs periodically in the transmission line. We consider an NOLM that consists of a $3 \mathrm{~dB}$-coupler, a highly nonlinear fiber (HNLF) and an attenuator inside the loop for unbalancing the NOLM. The output power of the NOLM is related to the input power by the relation

$$
P_{\text {out }}=\frac{G \eta \Delta}{2}\left\{1-\cos \left[\frac{G(1-\Delta)}{2} \sigma L_{\text {eff }} P_{\text {in }}\right]\right\} P_{\text {in }},
$$

where $\Delta$ is the attenuator value, $\eta=\exp \left(-\alpha L^{\prime}\right)$ represents the loss inside the loop, $L^{\prime}$ is the length of HNLF and $L_{\text {eff }}=(\eta-1) / \alpha$ is the effective length of the loop. Parameters $\sigma$ and $\alpha$ denote the nonlinearity and loss coefficients of the HNLF respectively. Parameter $G$ represents the amount of gain which is needed for an exact compensation of the losses inside the loop. Figure 1 shows different switching curves (obtained from Eq. 1) associated with the same operating point. The operating point is the closest point to the first switching peak such that $P_{\text {in }}=P_{\text {out }}$. We define parameter $s$ as the slope of the switching curve at the operating point. 


\section{ThB9}

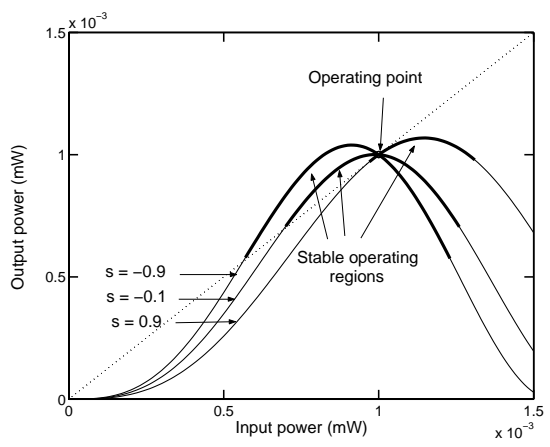

Fig.1 Switching curves of an NOLM (obtained from Eq. 1) for different value of parameter $s$ associated with the same operating point at $P=1 \mathrm{~mW}$. (a)

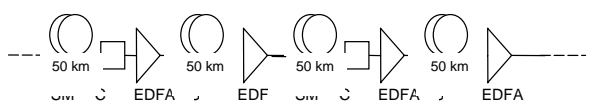

(b)

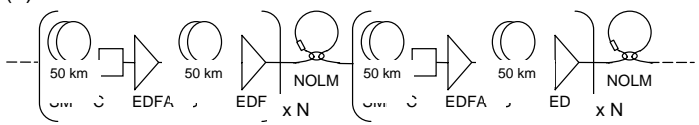

Fig.2 Schematic representation of the system (a) without NOLM, and (b) with NOLM.

Hence, for example, the curves in Fig. 1 corresponds to $s=-0.9,-0.1$ and +0.9 , respectively. A stable operating point is obtained for $-1<s<+1$. As the operating point is stable, any small input variation with respect to the operating power tends to be reduced at the output of the NOLM. As the maximum intensity of the 'ones' coincides with the operating point, this property is used to flatten the top of the pulses and reduce intersymbol interference (ISI). Furthermore, Fig. 1 indicates that when the input power approaches zero, the output power is attenuated. This property is exploited to reduce the growth of the side pulse structure.

\section{Results}

We consider a 10-Gb/s NRZ-transmission line which is schematically depicted in Fig. 2(a). The dispersion map length is $100 \mathrm{~km}$. Dispersion compensation is achieved by means of a lumped CFG located in the middle of the dispersion map. For the fiber, the dispersion coefficient is $17 \mathrm{ps} / \mathrm{km} / \mathrm{nm}$, the nonlinear coefficient is $1.4 \mathrm{~W}^{-1} \mathrm{~km}^{-1}$, and the loss coefficient is $0.22 \mathrm{~dB} / \mathrm{km}$. The CFGs have dispersion of $2130 \mathrm{ps}^{2}$ such that the average dispersion of the system is zero. The amplifier spacing is $50 \mathrm{~km}$. The amplifier gain is $10 \mathrm{~dB}$ and the noise figure is $4.5 \mathrm{~dB}$. Here, we compare the system without [Fig. 2(a)] and with [Fig. 2(b)] NOLMs . Input signal is a $10-\mathrm{Gb} / \mathrm{s}$ NRZ-pulses stream with raised cosine pulse shape with a bit-length of 100 ps. Both rise and fall times are equal to $40 \mathrm{ps}$ and the input modulation background is $20 \%$ of the average input power. Input data is a $2^{7}-1$ PRBS. All the NOLMs are identical for the given input power of the transmission line. According to the variations of the input power of the transmission line, the attenuator parameter and the loop length of the NOLMs have been carefully tuned such that the maximum power coincides with the operating point of the NOLM. As the average data power varies from $-2.7 \mathrm{dBm}$ to $8.5 \mathrm{dBm}$, the typical range for the attenuator parameter is about 15 to $25 \mathrm{~dB}$, while the loop length remains about few hundreds of meters. For the HNLF, the dispersion is assumed to be zero, the nonlinear coefficient is $12.7 \mathrm{~W}^{-1} \mathrm{~km}^{-1}$, and loss coefficient is $0.48 \mathrm{~dB} / \mathrm{km}$. Note that the gain of every amplifier which is located just before an NOLM in the transmission line has been increased up to $30 \mathrm{~dB}$ for compensation of the additional losses inside the NOLM. In this study, the random variations in the ripples parameters along the transmission line have been considered. We assume a normal distribution for the period $2 \pi / T_{0}$ and phase $\theta$ of the ripples. Mean value of the ripples period is $\left\langle 2 \pi / T_{0}\right\rangle=0.0641 \mathrm{~nm}(8 \mathrm{GHz})$ with a standard deviation of $0.002 \mathrm{~nm}$ $(0.25 \mathrm{GHz})$. The mean value of the ripples phase is $\langle\theta\rangle=\pi$ rad with a standard deviation equal to 0.34 rad. Figure 3 shows the evolution of the transmission performance in the grating-compensated DM fiber system which is represented in Fig. 2(a). The Q-factors at $4000 \mathrm{~km}$ are represented as a function of the input average powers for different values of the ripple amplitudes. The circles correspond to the case when no ripples are considered. The crosses, squares and triangles correspond to a ripple amplitude of 10 ps, 20 ps and $30 \mathrm{ps}$, respectively. Figure 3 shows that the ripples dramatically affect the transmission performance in the grating-compensated DM fiber system since the Q-factor is dramatically reduced when the ripple amplitude is increased. Figure 4 represents the evolution of transmission performance when the NOLMs are inserted in the transmission line, as it is represented in Fig. 2(b). We considered the case when the NOLM spacing is $500 \mathrm{~km}$ and the ripples amplitude is $20 \mathrm{ps}$. The Q-factors are represented as a function of the input average powers for different values of the slope of the switching curve $s$. For comparison, we represented the case when no NOLMs are in the system. Figure 4 indicates that a significant improvement of the system performance is achieved by means of NOLMs. It also demonstrates that the system including NOLMs has a 


\section{ThB9}

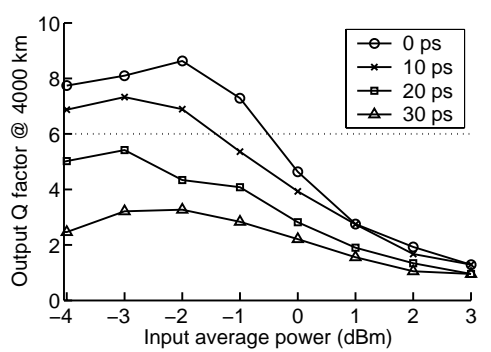

Fig. 3 Evolution of $\mathrm{Q}$ factor as a function of the input average power in the system without NOLMs for different values of the ripples amplitude.

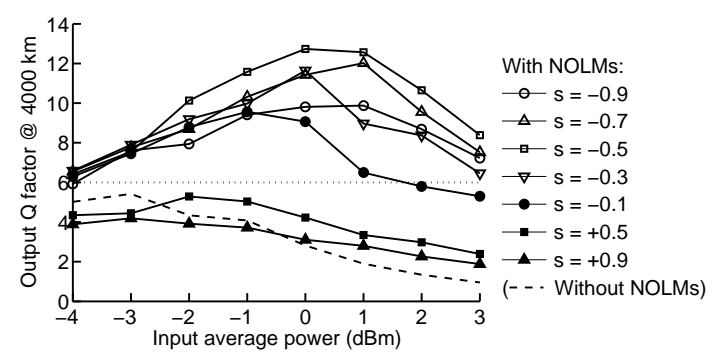

Fig.4 Evolution of Q factor as a function of the input average power in the system including NOLMs for different values of the parameter $s$. NOLM spacing is $500 \mathrm{~km}$ and ripples amplitude is $20 \mathrm{ps}$.
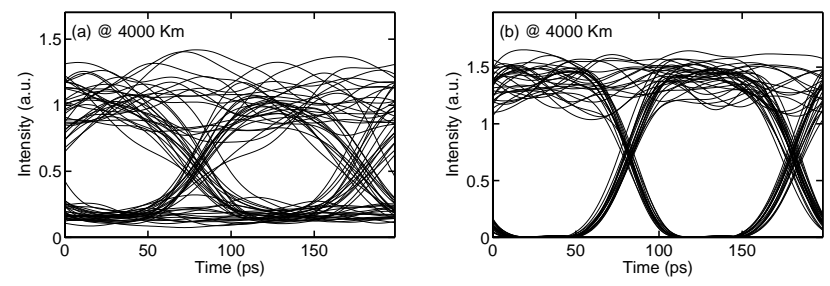

Fig.5 (a) Eye diagram at 4,000 km without NOLM, (b) Eye diagram at 4,000 km with NOLMs. The NOLM spacing is 500 $\mathrm{km}$ and ripples amplitude is $20 \mathrm{ps}$.

wide range of operating conditions with respect to the input powers. We found that the improvement caused by NOLM is strongly dependent on the value of the parameter $s$ and the optimum performance is obtained for $s=-0.5$. Note that no significant improvement is obtained when $s>0$. The main contribution of the NOLMs is to flatten the intensity of the 'ones' and remove the radiative background in the 'zeros' including amplifier noises and side peaks induced by the ripples. As $s$ ranges between +1 and -1 , stability around the operating power is re-enforced such that the NOLMs stabilize input pulses with large intensity fluctuations on their top. But, switching efficiency decreases with the value of $s$ leading to incomplete suppression of the side peaks in the 'zeroes.' Hence, the choice of parameter $s$ depends on the trade-off between the switching efficiency and stability. Figures 5 represent the eye diagrams at 4,000 $\mathrm{km}$ for the system without NOLMs [Fig. 5(a)] and with NOLMs [Fig. 5(b)]. Figure 5(b) clearly indicates that the action of NOLMs provide background suppression and amplitude flattening.

\section{Conclusion}

To conclude, we have investigated the use of NOLMs in 10-Gb/s NRZ grating-compensated DM transmission systems to reduce the impact of GDR. We have demonstrated that the transmission performance of the DM fiber system is dramatically improved by the action of the NOLMs. We have also reported that the transmission system incorporated with NOLMs has a wide range of operating condition for the input power. The proposed method provides a simple and economic way of upgrading terrestrial systems.

The authors acknowledge the support from the Research Grant Council of the Hong Kong Special Administrative Region, China, under Project PolyU5242/03E.

\section{References}

1. Y. H. C. Kwan et al., "Gaussian pulse propagation in dispersion-managed systems using chirped fiber gratings with group delay ripples," IEEE Photon. Technol. Lett. 17, 1025 (2005).

2. C. Scheerer et al., "System impact of ripples in grating group delay," ICTON'99, 33 (1999).

3. S. Boscolo et al, "Autosoliton transmission in dispersion-managed systems guided by in-line nonlinear optical loop mirrors," Opt. Lett., 25, 1240 (2000).

4. F. Seguineau et al., 'Experiment demonstration of simple NOLM-based 2R regenerator for 42.66 Gbit/s WDM long-haul transmissions," OFC'2004, Paper WN4 (2004). 